\title{
Rotation and instabilities for isotope and mass separation
}

\author{
J.-M. $\operatorname{Rax}^{1}$ and R. Gueroult ${ }^{2,3, \dagger}$ \\ ${ }^{1}$ Departments of Physics, University of Paris XI \& Ecole Polytechnique, LOA-ENSTA Chemin de la \\ Hunière, 91128 Palaiseau, France \\ ${ }^{2}$ Princeton Plasmas Physics Laboratory, Princeton University, Princeton, NJ 08540, USA \\ ${ }^{3}$ Plasma and Energy Conversion Laboratory (LAPLACE), Université de Toulouse, CNRS, INPT, UPS, \\ 31062 Toulouse, France
}

(Received 14 July 2016; revised 19 September 2016; accepted 20 September 2016)

Rotating plasmas have the potential to offer unique capabilities for isotope and mass separation. Among the various electric and magnetic field configurations offering mass separation capabilities, rotating plasmas produced through static or oscillating fields are shown to be a leading candidate for tackling the unsolved problem of large-scale plasma separation. The successful development and deployment of industrial-scale plasma separation technologies could, among many other applications, provide an innovative path towards advanced sustainable nuclear energy. In this context, the potential and versatility of plasma rotation induced by rotating magnetic fields is uncovered and analysed. Analytical stability diagrams are derived from rotating ion orbits as a function of ion mass. Based on these findings, the basic principles of a rotating field plasma separator are then introduced. In light of these results, challenges associated with this original separation process are underlined, and the main directions for a future research program aimed at this important unsolved problem of applied plasma physics are identified.

Key words: magnetized plasmas, plasma applications, plasma confinement

\section{Introduction}

Among the various unsolved problems of plasma physics, controlled thermonuclear fusion remains the most challenging one, both with respect to its complexity and to the forthcoming critical energy transition. All projections of future energy consumption converge towards a doubling, at the very least, of the world's energy demand by the end of this century. Today's consumption is approximately $10^{20} \mathrm{~J} \mathrm{yr}^{-1}$. Given the increasing evidence of the dramatic impact of oil and coal energy sources on our environment, thermonuclear fusion and advanced nuclear fission are the only energy sources providing a straightforward development path toward a long term, sustainable, post-industrial society for which the energy demand is foreseen to, at least, double.

Nuclear fission, in particular with the deployment of fourth generation fast breeders, has the potential to ensure a soft energy transition. However, the management of

$\dagger$ Email address for correspondence: rgueroul@pppl.gov 
nuclear fuel cycle remains an open problem. Further to nuclear fusion, plasma physics and technologies could provide novel and unique solutions in this context as well. Indeed, plasmas offer a wide range of possibilities for isotope and mass separation (Grossman \& Shepp 1991; Timofeev 2007, 2014; Gueroult, Hobbs \& Fisch 2015). However, despite experimental demonstrations in the form of plasma centrifugation (Krishnan, Geva \& Hirshfield 1981; Hole \& Simpson 1999), ion cyclotron resonance (Dawson et al. 1976; Dolgolenko \& Muromkin 2009) and the historical calutron configuration; plasma isotope and mass separation are not to date used at industrial scale. In this respect, plasma separation remains an unsolved problem of plasma physics.

Plasma separation could be particularly useful for reprocessing burned nuclear fuel and ashes, with the objective of closing the nuclear cycle (Timofeev 2007; Gueroult $\&$ Fisch 2014). Two choices can be made with nuclear energy. First, an open cycle, where the downloaded burned fuel is not reprocessed. Second, a closed cycle, where reprocessing provides new fuel, both as fissile and fertile materials, and reduces the volume of high-level waste, particularly through the separation of minor actinides (Timofeev 2007; Gueroult \& Fisch 2014).

It is commonly accepted that closing the nuclear cycle with the fourth generation of fast breeders would increase the energy extracted from uranium by a factor of approximately 50 to 60 .

Closed cycle reprocessing is generally based on hot chemistry with a large number of steps in large-scale facilities. Besides the inventory problem associated with the large number of steps, particularly for minor actinides, huge volumes of organic solvent and acids are used for extraction. In addition, large volumes of nuclear waste are transported, for separation and then storage, through the countries who made the closed cycle choice (France, Japan, China, Russia and the UK). In contrast, plasma separation has the potential to offer efficient reprocessing in a single step. A small footprint plasma based facility located near each nuclear power station, providing in situ efficient reprocessing, hence appears particularly attractive for the development of advanced nuclear energy.

Each ${ }^{235} \mathrm{U}$ fission releases an energy of the order of $200 \mathrm{MeV}$, and the ratio ${ }^{235} \mathrm{U} /{ }^{238} \mathrm{U}$ is of the order of a few per cent. Each nucleus in the irradiated nuclear fuel to be processed is thus associated with an energy deposited in the reactor core of the order of a few MeV. Plasma reprocessing cost can be roughly estimated based on the following assumptions. First, each atom of the burned fuel will have to be ionised. This ionisation cost is a few tens of eV per atom. Second, an electron temperature of the order of $10 \mathrm{eV}$ has to be maintained to ensure suitable plasma parameters. Third, a selected minority of ions have to be accelerated or heated to be separated. Corresponding energy losses are expected to be up to a few $100 \mathrm{eV}$ per selected ion. Fourth, the background ion temperature will be brought from room temperature to a fraction of an $\mathrm{eV}$. Summing up these contributions, an energy cost of a few $\mathrm{keV}$ per atom seems like a safe upper bound for the whole plasma process. With these estimates, the power requirement to achieve a closed fuel cycle with plasma separation technologies will be below one per cent of the reactor power, far below the projected gain of 50-60.

In addition to this favourable basic power balance, estimates of plasma processing throughput are consistent with the need of a typical fission reactor. Typical ion current density in plasmas is given by the relation $n C_{s}$ where $C_{s}$ is the ion acoustic velocity, $C_{s} \sim 10^{5} \mathrm{~cm} \mathrm{~s}^{-1}$ and $n$ the plasma density. In advanced helicons and Electron Cyclotron Resonance (ECR) plasma sources, plasma density 
can be as large as $10^{12}-10^{13} \mathrm{~cm}^{-3}$. The continuous processing of a beam with cross-section $S \sim 10^{3} \mathrm{~cm}^{2}$ during one year, that is to say $\tau \sim 10^{7} \mathrm{~s}$, is thus equivalent to approximately $n C_{s} S \tau \sim 10^{28}$ nuclei. This is to be compared with the $10^{24}$ nuclei contained in $1 \mathrm{~kg}$ of irradiated fuel. Based on these simple estimates, about $10^{4} \mathrm{~kg}$ of irradiated fuel can be processed per year. Interestingly, this is about the amount of burned fuel downloaded from a typical reactor per year.

Among the numerous separation mechanisms one can envision in a magnetised plasma column, classical electric field-driven centrifugation (Ohkawa \& Miller 2002; Rax et al. 2015), advanced magnetic centrifugal mass filter (Fetterman \& Fisch 2011a; Gueroult \& Fisch 2012; Gueroult, Rax \& Fisch 2014), ion cyclotron resonances (Dawson et al. 1976; Dolgolenko \& Muromkin 2009), auto-resonant ion cyclotron resonance (Rax, Robiche \& Fisch 2007) and wave-driven centrifugation (Fetterman \& Fisch 2009, 2011c) have been proposed and deemed promising for isotope and mass separation. In this study, after briefly reviewing rotating plasma separation schemes in the next section, we identify and analyse a new scheme: the use of a rotating magnetic field for isotope and mass separation in a plasma column.

That a rotating magnetic field should drag a fluid plasma rotation is a direct consequence of the Alfvén frozen in law, but (i) the dynamics at the single particle level is far more complex than a simple rotation (Soldatenkov 1966; Fisch \& Watanabe 1982; Hugrass \& Jones 1983), and (ii) each choice of different driving external currents, for the very same magnetic field, provide new classes of orbits. This unexplored versatility offers several pathways for optimisation. In $\S 2$, the different possible methods for spinning a magnetised plasma for the purpose of mass separation are reviewed. Moving on to rotating magnetic fields, in $\S 3$, three different rotating vector potentials providing the same magnetic field are introduced. The associated, external or internal, currents are analysed and shown to be physically realisable. Then in $\$ \S 4-6$ we present an analysis of the orbital stability for these three rotating configurations. The relative advantages and drawbacks of these configurations for plasma mass separation are discussed in $\$ 7$. Finally, $\$ 8$ gives a summary of these new results pertaining to one of the most challenging unsolved problem of plasma physics: isotope and mass separation in rotating plasmas.

\section{Angular momentum conversion and rotating plasmas for mass separation}

To assess the originality of rotating magnetic fields for isotope and mass separation, let us briefly review the principles of other rotating plasma configurations. As per usual in plasma physics, the analysis of the physical processes must be performed both at the macroscopic fluid level and at the microscopic single particle level. Isotope separation with rotating plasmas offers a clear illustration of this rule. At the fluid level the description can be reduced to a uniform rotation with a steady state vorticity. On the other hand, at the single particle level, the orbits always display more complex behaviours than a simple rotation, leading to the occurrence of orbital instabilities. These instabilities can in turn be put at work for mass discrimination. The macroscopic averaging of these mass-dependent complex orbits is responsible for the emerging simple uniform rotation at the fluid level. In this study we will concentrate on a microscopic analysis and on the identification of orbital instabilities under various rotating fields configurations.

The Brillouin limit associated with the rigid body rotation of a magnetised plasma column has been explored both theoretically and experimentally for nuclear waste 


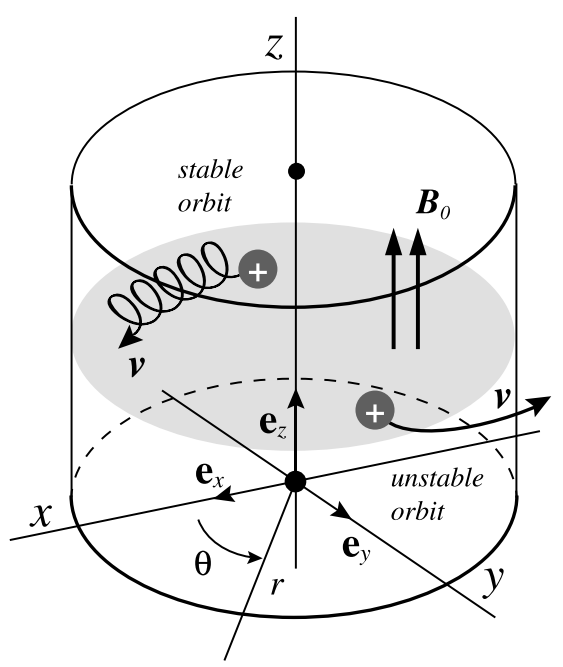

FIGURE 1. Classical axially magnetised plasma configuration for mass separation. The azimuthal rotation can be induced through any angular momentum deposition process around the $z$ axis.

management. Consider an ion, with mass $m$ and charge $q$, interacting with a static radial linear electric field $\boldsymbol{E}$ and an axial uniform magnetic field $\boldsymbol{B}_{0}$

$$
\frac{q}{m}\left[\boldsymbol{E}(x, y), \boldsymbol{B}_{0}\right]=\left[\Omega_{E}^{2} x \boldsymbol{e}_{x}+\Omega_{E}^{2} y \boldsymbol{e}_{y}, \Omega_{c} \boldsymbol{e}_{z}\right],
$$

as depicted in figure 1. Here, $\left[\boldsymbol{e}_{x}, \boldsymbol{e}_{y}, \boldsymbol{e}_{z}\right]$ is a Cartesian basis and $[x, y, z]$ is the associated Cartesian coordinates. In this configuration, ion orbits are radially confined if the Brillouin condition (Rax et al. 2015),

$$
\Omega_{c}>\sqrt{2} \Omega_{E},
$$

is fulfilled. For $\Omega_{c}<\sqrt{2} \Omega_{E}$ ions are ejected radially from the plasma column. As the cyclotron frequency is given by $\Omega_{c}=q B_{0} / m$ and $\Omega_{E} \sim 1 / \sqrt{m}$, for a given configuration, the heavy elements are expelled from a rotating plasma mixture and the light ones remain confined. It is to be noted that this simple configuration can be used in another way if we sustain an electrostatic potential with two radial minima rather than one with a parabolic potential associated with a linear field (Gueroult et al. 2014). Beside these two simple $E$ cross $B$ configurations, more sophisticated fields configurations have been identified and analysed for isotope processing. The auto-resonant interaction ( $\operatorname{Rax}$ et al. 2007) between an ion beam with axial velocity $V$ and the helical magnetic field associated with the vector potential $\boldsymbol{A}$,

$$
\frac{q}{m} \boldsymbol{A}(x, y, z)=\left[\frac{q A}{m} \cos \int_{0}^{z} k(u) \mathrm{d} u-\frac{\Omega_{c}}{2} y\right] \boldsymbol{e}_{x}+\left[\frac{q A}{m} \sin \int_{0}^{z} k(u) \mathrm{d} u+\frac{\Omega_{c}}{2} x\right] \boldsymbol{e}_{y},
$$

also provides a very efficient separation process if the periodic transverse field, with local wave vector $k(z)$, is tapered according to the cyclotron auto-resonance relation

$$
k(z) V=\Omega_{c}\left[\cos \left(\frac{2 q A \Omega_{c}}{m V^{2}} z+\sum_{n=1}^{+\infty} \frac{\mathrm{J}_{n}(n)}{n} \sin \left(\frac{4 n q A \Omega_{c}}{m V^{2}} z-n \pi\right)\right)\right]^{-1} .
$$


Here, $\mathrm{J}_{n}$ is the ordinary Bessel function of order $n$. Such a tapered helical wiggler configuration ensures that the resonant conversion of the linear momentum $m V$, along $z$, into an angular momentum, around $z$, is only efficient for a given mass, and inefficient for all other masses.

In between the simple radial $E$ cross axial $B$ configurations, and this complex tapered helical wiggler configuration, a variety of electric and magnetic geometries and topologies have been studied, and should be explored in order to solve the open problem of plasma isotope and mass separations. Another very elegant and efficient way to sustain plasma rotation was recently identified and analysed (Fetterman \& Fisch 2011c): angular momentum injection through resonant waves-particle interactions. A cylindrical plasma wave whose electric (and/or magnetic) potential $\Phi$ varies according to

$$
\Phi(x, y, z, t)=\phi(r) \cos (l \theta+k z-\omega t),
$$

with $r^{2}=x^{2}+y^{2}$ and $\theta$ the polar coordinates (as illustrated in figure 1), $l$ an integer and $\phi(r)$ an appropriate solution of the radial Maxwell equations, interacting with a cylindrical plasma column, is able to transfer to magnetised particles (i) energy, (ii) axial linear momentum along $z$, and (iii) azimuthal angular momentum around $z$. For this transfer to happen, the velocity $V=\mathrm{d} z / \mathrm{d} t$ of the particles must satisfy the Doppler shifted cyclotron resonance condition, $k V=n \Omega_{c}+\omega$ where $n$ is any integer. If an amount of energy $\delta W$ is transferred from this wave to the particles, an amount of axial linear momentum $\delta \boldsymbol{P}=m \delta V \boldsymbol{e}_{z}$ and an amount of angular momentum $\delta \boldsymbol{L}$ appear in the plasma,

$$
\begin{aligned}
\delta \boldsymbol{P} & =\frac{k}{\omega} \delta W \boldsymbol{e}_{z}, \\
\delta \boldsymbol{L} & =\frac{l}{\omega} \delta W \boldsymbol{e}_{z} .
\end{aligned}
$$

The plasma column will respond to this linear momentum deposition through a $z$ acceleration and to this angular momentum deposition through a rotation. The energy deposition profile $\delta W(r)$, the collisional dissipation and the plasma column inertia tensor will determine the value of the angular velocity.

The frozen in law with a transverse rotating magnetic field $\boldsymbol{b}(t)$

$$
\frac{q}{m} \boldsymbol{b}(t)=\omega_{c} \cos \Omega t \boldsymbol{e}_{x}+\omega_{c} \sin \Omega t \boldsymbol{e}_{y},
$$

provides yet another original mechanism to spin a magnetised plasma column around the $z$ axis. The potential of such a configuration for mass separation will be presented and analysed here. Compared to current generation applications (see, for example, Fisch \& Watanabe (1982)) for which $\Omega$ is chosen such that $\omega_{c i}<\Omega<\omega_{c e}$ to drive an azimuthal electron current, the regime envisioned here is the one for which the entire plasma column (both ions and electrons) rotates, with zero or limited associated azimuthal current. The realisation of this new configuration for mass separation is not straightforward because a magnetised plasma column will always accept a radial static electric field $\boldsymbol{E}(x, y)$ strictly perpendicular to the static magnetic field $\boldsymbol{B}_{0}$ or a plasma wave belonging to one of its dispersion branches, but it will always screen out a time varying magnetic field $\boldsymbol{b}(t)$. The screening length depends on collisionality and is in between the collisionless and diffusive limits $\lambda_{L}=c / \omega_{p e}$ and $\lambda_{K}=\left(\mu_{0} \sigma \omega\right)^{-1 / 2}$, which are respectively the London and Kelvin characteristic lengths. Here, $\omega_{p e}$ is the plasma frequency, $c$ is the speed of light, $\mu_{0}$ is the vacuum permittivity, $\sigma$ is the plasma electric conductivity and $\omega$ is the wave frequency. Since the potential of this type of 
(a)

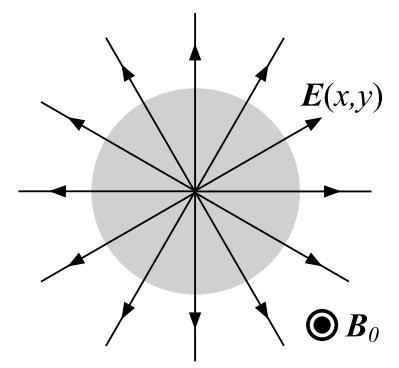

Crossed fields drift (b)

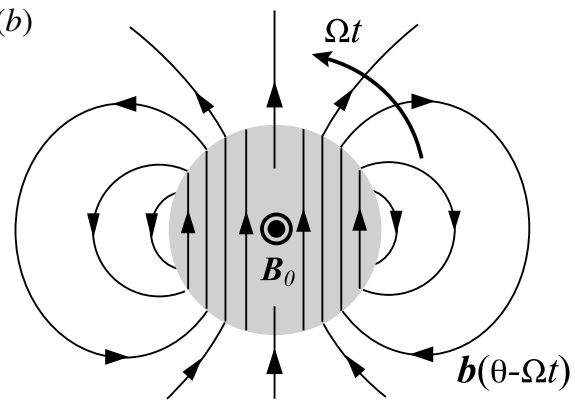

Rotating magnetic field (c)

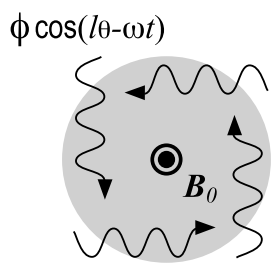

Rotating wave

FIGURE 2. Angular momentum injection in an axially magnetised plasma column, (a) $\boldsymbol{E}(x, y)$ crossed fields drift, $(b)$ rotating $\boldsymbol{b}(\theta-\Omega t)$ magnetic field, $(c)$ rotating cylindrical $\phi \cos (l \theta-\omega t)$ wave.

rotating plasma configuration for mass separation and nuclear waste management has never been explored, we will analyse the orbit at the single particle level, for various schemes, to evaluate the potential for mass separation.

To conclude this discussion, let us summarise, and illustrate in figure 2 , an emerging classification of the physical processes aimed at rotating an axially magnetised plasma for the purpose of isotope and mass separations: (i) the $E$ cross $B$ drift with a linear radial electric field (see figure $2 a$ ), (ii) the use of the frozen in law with a rotating magnetic field $\boldsymbol{b}(t)$ (see figure $2 b$ ), (iii) direct angular momentum absorption from a cylindrical plasma wave displaying a high content of angular momentum (see figure $2 c$ ). In this paper, we will consider three basic types of rotating magnetic fields configurations leading to the same rotating magnetic field $\boldsymbol{b}(t)$ and focus the analysis on the stability of ion orbits for the purpose of isotope and mass separation. We will demonstrate that, for appropriate field strength and frequency, two neighbouring masses display completely different behaviours, one performing a stable multi-periodic oscillation near the $z$ axis and the other being expelled radially far from the $z$ axis.

\section{The three basic rotating fields configurations}

Plasma interaction with rotating magnetic fields has been investigated in the past for the purpose of thermonuclear confinement (Soldatenkov 1966; Hugrass \& Jones 1983), current generation (Fisch \& Watanabe 1982) and plasma acceleration (Shinohara et al. 2014), but the potential for separation technologies has never been considered.

Consider a homogeneous magnetic field $\boldsymbol{b}(t)$ rotating at an angular velocity $\Omega$,

$$
\boldsymbol{b}(t)=\frac{m}{q} \omega_{c}\left(\cos \Omega t \boldsymbol{e}_{x}+\sin \Omega t \boldsymbol{e}_{y}\right) .
$$

This magnetic field can be derived from any linear combination of the following vector potentials: (i) $\boldsymbol{A}_{s}$ and $\boldsymbol{A}_{a}$ or (ii) $\boldsymbol{A}_{s}$ and $\boldsymbol{A}_{c}$ or (iii) $\boldsymbol{A}_{c}$ and $\boldsymbol{A}_{a}$.

$$
\begin{aligned}
\frac{q}{m} \boldsymbol{A}_{s} & =\omega_{c}(y \cos \Omega t-x \sin \Omega t) \boldsymbol{e}_{z}, \\
\frac{q}{m} \boldsymbol{A}_{a} & =\omega_{c} z\left(\sin \Omega t \boldsymbol{e}_{x}-\cos \Omega t \boldsymbol{e}_{y}\right), \\
\frac{q}{m} \boldsymbol{A}_{c} & =\frac{\omega_{c}}{2}\left[z\left(\sin \Omega t \boldsymbol{e}_{x}-\cos \Omega t \boldsymbol{e}_{y}\right)+(y \cos \Omega t-x \sin \Omega t) \boldsymbol{e}_{z}\right] .
\end{aligned}
$$


To each of these three magnetic vector potentials corresponds a different electric field $\boldsymbol{E}=-\partial \boldsymbol{A} / \partial t$. For completeness, these electric fields read

$$
\begin{aligned}
\frac{q}{m} \boldsymbol{E}_{s} & =\omega_{c} \Omega(x \cos \Omega t+y \sin \Omega t) \boldsymbol{e}_{z}, \\
\frac{q}{m} \boldsymbol{E}_{a} & =-\omega_{c} \Omega z\left(\cos \Omega t \boldsymbol{e}_{x}+\sin \Omega t \boldsymbol{e}_{y}\right), \\
\frac{q}{m} \boldsymbol{E}_{c} & =-\frac{\omega_{c}}{2} \Omega\left[z\left(\cos \Omega t \boldsymbol{e}_{x}+\sin \Omega t \boldsymbol{e}_{y}\right)-(x \cos \Omega t+y \sin \Omega t) \boldsymbol{e}_{z}\right] .
\end{aligned}
$$

The rotating magnetic field configuration is thus not unique and must be viewed as a two-dimensional vector space, but the orbit in a given combination of $\boldsymbol{A}_{s}$ and $\boldsymbol{A}_{a}$ is not a simple combination of the orbit in $\boldsymbol{A}_{s}$ and $\boldsymbol{A}_{a}$. This is a simple consequence, as it will become clear, of the fact that the determinant of the sum of two matrices is different from the sum of their determinants. It is worth noting that most of the plasma literature on rotating magnetic fields for thermonuclear confinement or current generation is restricted to $\boldsymbol{A}_{s}$, while the simplest configuration is arguably the one described by $\boldsymbol{A}_{c}$, which corresponds to two phased coils. These three vector potentials, $\boldsymbol{A}_{s}, \boldsymbol{A}_{a}$ and $\boldsymbol{A}_{c}$, cannot be reduced to the same function through a gauge transformation and these different expressions are associated with different boundary conditions, that is to say with different external and plasma currents.

$\boldsymbol{A}_{s}$ is the most popular expression and can be produced with an axial surface current on a cylinder aligned along $z$. The current amplitudes are proportional to $\cos (\theta-\Omega t)$ where $\theta$ is the polar angle around the $z$ axis. $\boldsymbol{A}_{a}$ is the vector potential describing the vicinity of the electric node of an $l=1$ standing surface Alfvén wave along a cylindrical plasma column, where $l$ is the Fourier mode number associated with exp $j l \theta$. Finally, $\boldsymbol{A}_{c}$ is the vector potential describing the classical synchronous rotating field generated by two coils whose axes are oriented along $x$ and $y$ and whose currents are out of phase by an angle $\pi / 2$.

These three field configurations $\boldsymbol{A}_{s}, \boldsymbol{A}_{a}$ and $\boldsymbol{A}_{c}$ provide a convenient and tractable theoretical framework to illustrate mass separation effects in rotating magnetic fields. However, these configurations only describe an idealised plasma. Practically, various effects could limit the range of plasma parameters and device dimensions for which the simple picture derived in this paper will hold true. Although the full study of these practical effects is beyond the scope of this paper, possible challenges towards the implementation of these three configurations are briefly discussed for completeness in the remaining of this section.

First, $\boldsymbol{A}_{a}$ appears a priori to be the best candidate since it is a normal mode of a plasma mixture. As a result, it does not suffer from plasma screening. However, the main limitation of this configuration stems from the fact that $\boldsymbol{A}_{a}$ only describes the local field of a standing wave in the vicinity of an electric node of this wave. The full structure of the vector potential of a surface Alfvén wave, with wave vector $k_{A}$ along a plasma cylinder, is given by $\boldsymbol{A}_{a}^{*}$,

$$
\frac{q}{m} \boldsymbol{A}_{a}^{*}=\frac{\omega_{c}}{k_{A}}\left[\sin \left(k_{A} z\right) \sin (\Omega t) \boldsymbol{e}_{x}-\sin \left(k_{A} z\right) \cos (\Omega t) \boldsymbol{e}_{y}\right] .
$$

A full analysis of ion orbit stability would thus require considering $\boldsymbol{A}_{a}^{*}$ in lieu of the idealised vector potential $\boldsymbol{A}_{a}$ used in this study.

Then, $\boldsymbol{A}_{c}$ is to be considered because of the simplicity of the forcing currents configuration. However, since it is not a normal mode of the plasma, penetration 
of the rotating field within the plasma column will depend on plasma parameters, and $\boldsymbol{A}_{c}$ only describes the asymptotic limit corresponding to instantaneous field penetration. Field penetration for similar coil configurations has been shown to be a strong function of two dimensionless parameters (Milroy 1999): (i) the plasma column radius $a$ normalised by the Kelvin characteristic length $\lambda_{K}$ and (ii) the electron cyclotron frequency - computed for the rotating magnetic field - normalised by the electron-ion collision frequency. For large devices $\left(a \gg \lambda_{K}\right)$, electron-ion collisionality has to be sufficiently small to ensure fast field penetration. For mass separation purposes, this would translate into a trade-off between plasma density, plasma dimensions and magnetic field intensity.

Similarly, $\boldsymbol{A}_{s}$ is not a normal mode of the plasma column. In this configuration, electron screening is expected to take place because the inductive electric field $-\partial \boldsymbol{A}_{s} / \partial t$ is along the static field lines. For collisionless plasmas, one can solve Maxwell-Faraday and Maxwell-Ampere equations taking into account the response of the plasma electrons (mass $m_{e}$, charge $q_{e}$ ) along $z$ to find the screened vector potential $\boldsymbol{A}_{s}^{*}$ inside the plasma,

$$
\frac{q}{m} \boldsymbol{A}_{s}^{*}=\frac{\omega_{c}}{k_{p}}\left[\sinh \left(k_{p} y\right) \cos (\Omega t)-\sinh \left(k_{p} x\right) \sin (\Omega t)\right] \boldsymbol{e}_{z} .
$$

Here $k_{p}^{-1}=\lambda_{p}$ is the London length, or inertial skin depth, defined as $\lambda_{p}^{2}=m_{e} c^{2} \varepsilon_{0} / n q_{e}^{2}$ ( $c$ is the velocity of light and $\varepsilon_{0}$ the permittivity of vacuum). For plasma density $n \sim 10^{12} \mathrm{~cm}^{-3}$, the inertial skin depth is of the order of a centimetre. The rotating magnetic field will display a homogeneous structure near the $z$ axis, and an inhomogeneous one near the edge since $\cosh \left(x / \lambda_{p}\right)=1+x^{2} / 2 \lambda_{p}{ }^{2}+O\left(x^{4} / \lambda_{p}{ }^{4}\right)$ and $\cosh \left(y / \lambda_{p}\right)=1+y^{2} / 2 \lambda_{p}{ }^{2}+O\left(y^{4} / \lambda_{p}{ }^{4}\right)$. As a result, the effect of plasma screening on the orbit stability will mainly be important at the edge. Here again, a full analysis of ion orbit stability would require using $\boldsymbol{A}_{s}^{*}$ rather than the idealised vector potential $\boldsymbol{A}_{s}$.

Let us conclude this discussion of possible limitations with a practical observation. For minor actinides separation (Gueroult \& Fisch 2014), if we choose to expel the heavy part, the core will be cleaned up and the edge will be a mix of light and heavy ions. However, the study of the stability diagram, taking into account the nonlinear coupling terms associated with electron screening $\left(\boldsymbol{A}_{s}^{*}\right.$ in lieu of $\boldsymbol{A}_{s}$ ), will make it possible to modify the separation strategy using well-identified nonlinear stability boundaries. This identification is left for a future study, and so is the identification of the optimum combination of $\boldsymbol{A}_{s}$ and $\boldsymbol{A}_{c}$, both from the standpoint of orbits and from the standpoint of field penetration in the plasma. However, we note in passing that a $1 / 1$ combination of these vectors gives $\boldsymbol{A}_{a}$, which is a natural mode of the plasma. As a result, there is clearly a synergy for penetration between these vacuum modes.

In the following three sections, in order to address the open problem of mass separation with rotating magnetic fields, we will compare ion orbits in the combination of an axial static magnetic field $\boldsymbol{B}_{0}$ and a transverse rotating magnetic field $\boldsymbol{\nabla} \times \boldsymbol{A}$,

$$
\nabla \times \boldsymbol{A}+\boldsymbol{B}_{0}=\frac{m}{q} \omega_{c}\left(\cos \Omega t \boldsymbol{e}_{x}+\sin \Omega t \boldsymbol{e}_{y}\right)+\frac{m}{q} \Omega_{c} \boldsymbol{e}_{z},
$$

for the ideal potential vectors $\boldsymbol{A}=\boldsymbol{A}_{a}(\S 4), \boldsymbol{A}=\boldsymbol{A}_{c}$ (§ 5) and finally $\boldsymbol{A}=\boldsymbol{A}_{s}$ (§ 6). To analyse the orbits we will consider Newton's equations for an ion with mass $m$, charge $q$ and velocity $\boldsymbol{V}$,

$$
m \frac{\mathrm{d} \boldsymbol{V}}{\mathrm{d} t}=-q \frac{\partial \boldsymbol{A}}{\partial t}+q \boldsymbol{V} \times(\boldsymbol{\nabla} \times \boldsymbol{A})+q \boldsymbol{V} \times \boldsymbol{B}_{0},
$$


and calculate the stability diagram in the $\left(\Omega / \Omega_{c}, \omega_{c} / \Omega_{c}\right)$ space as $\Omega, \Omega_{c}$ and $\omega_{c}$ are the three control parameters of these innovative separation schemes. In the last sections, on the basis of these stability diagrams, we will consider the potential for mass separation. To simplify the forthcoming studies, we define the control parameters $u$ and $v$ and the normalised eigenfrequency $w$ as follows,

$$
\begin{aligned}
& u=\frac{\Omega}{\Omega_{c}}, \\
& v=\frac{\omega_{c}}{\Omega_{c}}, \\
& w=\frac{\omega}{\Omega_{c}},
\end{aligned}
$$

where $\omega$, through a factor $\exp j \omega t$, will be the generic pulsation of the ion periodic motion. The position of $w$ in the complex plane, as a function of $(u, v)$, will decide of the stability, or the instability, of the orbit.

\section{Orbital stability with a surface Alfvén standing wave}

Consider a standing low-frequency wave along a magnetised plasma column belonging to the Alfvénic branch of the dispersion relation, the vector potential for an $l=1$ surface wave is given by $\boldsymbol{A}_{a}$ in the vicinity of an electric node. Then, let us write down the dynamics of an ion, with mass $m$ and charge $q$, on a Cartesian set of axis where $z$ is oriented along the static magnetic field $\boldsymbol{B}_{0}$ :

$$
\begin{aligned}
\frac{\mathrm{d}^{2} x}{\mathrm{~d} t^{2}} & =\Omega_{c} \frac{\mathrm{d} y}{\mathrm{~d} t}-\omega_{c} \frac{\mathrm{d} z}{\mathrm{~d} t} \sin \Omega t-\omega_{c} \Omega z \cos \Omega t, \\
\frac{\mathrm{d}^{2} y}{\mathrm{~d} t^{2}} & =-\Omega_{c} \frac{\mathrm{d} x}{\mathrm{~d} t}+\omega_{c} \frac{\mathrm{d} z}{\mathrm{~d} t} \cos \Omega t-\omega_{c} \Omega z \sin \Omega t, \\
\frac{\mathrm{d}^{2} z}{\mathrm{~d} t^{2}} & =\omega_{c} \frac{\mathrm{d} x}{\mathrm{~d} t} \sin \Omega t-\omega_{c} \frac{\mathrm{d} y}{\mathrm{~d} t} \cos \Omega t .
\end{aligned}
$$

We recognise a total time derivative in the first and second equation, so the order of the first two equations can be lowered. We introduce the fixed guiding centres $\left(x_{g}, y_{g}\right)$ of the transverses oscillations and rewrite these two equations

$$
\begin{aligned}
& \frac{\mathrm{d} x}{\mathrm{~d} t}-\Omega_{c}\left(y-y_{g}\right)+\omega_{c} z \sin \Omega t=0, \\
& \frac{\mathrm{d} y}{\mathrm{~d} t}+\Omega_{c}\left(x-x_{g}\right)-\omega_{c} z \cos \Omega t=0 .
\end{aligned}
$$

We move from the laboratory frame $(x, y)$ to a rotating frame $(X, Y)$ through the transformation

$$
\left(x-x_{g}\right)+j\left(y-y_{g}\right)=(X+j Y) \exp j \Omega t,
$$

and we get the following set of relations describing the dynamics in this rotating frame

$$
\begin{aligned}
\frac{\mathrm{d} X}{\mathrm{~d} t}-\left(\Omega+\Omega_{c}\right) Y & =0, \\
\frac{\mathrm{d} Y}{\mathrm{~d} t}+\left(\Omega+\Omega_{c}\right) X-\omega_{c} z & =0, \\
\frac{\mathrm{d}^{2} z}{\mathrm{~d} t^{2}}+\Omega \omega_{c} X+\omega_{c} \frac{\mathrm{d} Y}{\mathrm{~d} t} & =0 .
\end{aligned}
$$




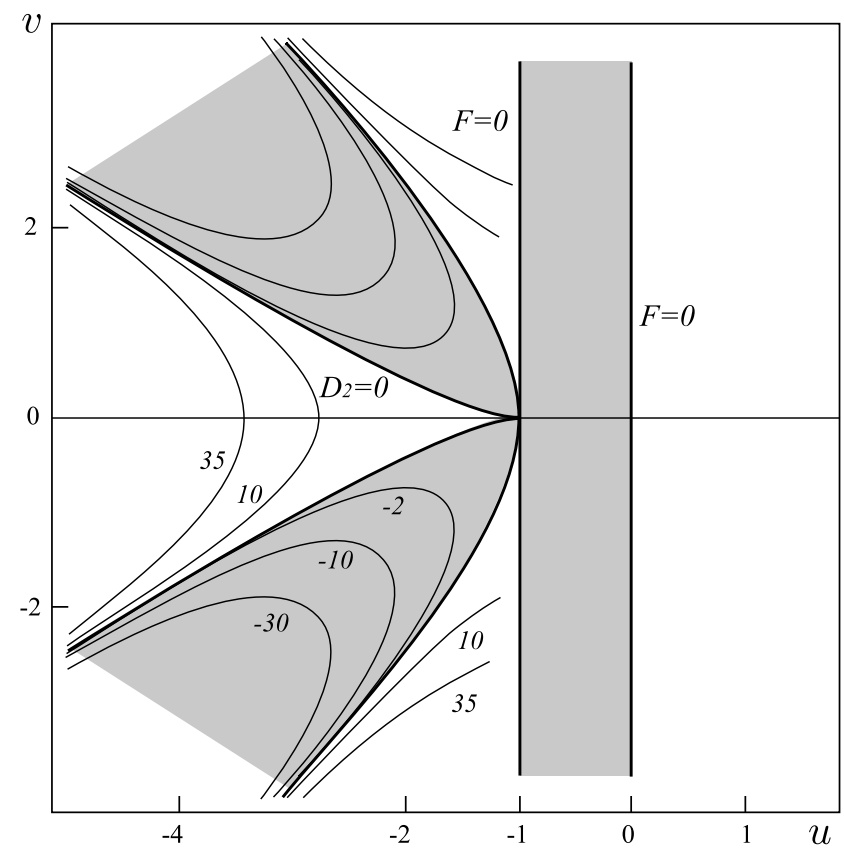

FIgURE 3. Stability regions, $D_{2}>0, F>0$, in the $u=\Omega / \Omega_{c}$ and $v=\omega_{c} / \Omega_{c}$ plane, for ion orbits in a surface Alfvén standing wave, the contour lines are labelled with the value of $D_{2}(u, v)$.

A normal mode analysis can be performed as these equations are linear and the coefficients are constant. Thus, we define the amplitudes $\left(\boldsymbol{X}_{0}, Y_{0}, \boldsymbol{z}_{0}\right)$ of the multi-periodic motion,

$$
[X(t), Y(t), z(t)]=\left(\boldsymbol{X}_{0}, \boldsymbol{Y}_{0}, \boldsymbol{z}_{0}\right) \exp j \omega t .
$$

Introducing the control parameters $(u, v)$ and the normalised eigenfrequency $w=\omega / \Omega_{c}$, the characteristic equation, resulting from the nullity of the characteristic determinant, displays the simple form

$$
w^{4}-\left((1+u)^{2}+v^{2}\right) w^{2}+u v^{2}(1+u)=0 .
$$

For stability, the discriminant $D_{2}(u, v)$ of the associated algebraic second-order equation with unknown $w^{2}$ must be positive and the two real roots must be positive and different. Two numbers are strictly positive if and only if their sum and product are strictly positive, so the stability condition, $\omega=w \Omega_{c}$ real, in the $(u, v)$ plane reduces to

$$
\begin{aligned}
D_{2}(u, v) & =(1+u)^{4}+2\left(1-u^{2}\right) v^{2}+v^{4}>0, \\
F(u) & =u(1+u)>0 .
\end{aligned}
$$

The shaded regions in figure 3 are associated with unstable orbits and the contour lines indicate the value of $D_{2}(u, v)$ which is proportional to the square of the growth rate of the exponential instability of the unconfined orbits. 


\section{Orbital stability with dephased orthogonal coils}

Let us consider two solenoidal coils whose axes are oriented along $x$ and $y$, in the vicinity of the origin $x=y=z=0$, the superposition of their vector potentials is given by $\boldsymbol{A}_{c}$. The dynamics of an ion on a Cartesian set of axis, where $z$ is oriented along the static magnetic field $\boldsymbol{B}_{0}$, is given by the solutions of

$$
\begin{gathered}
\frac{\mathrm{d}^{2} x}{\mathrm{~d} t^{2}}=\Omega_{c} \frac{\mathrm{d} y}{\mathrm{~d} t}-\omega_{c} \frac{\mathrm{d} z}{\mathrm{~d} t} \sin \Omega t-\frac{\Omega \omega_{c}}{2} z \cos \Omega t, \\
\frac{\mathrm{d}^{2} y}{\mathrm{~d} t^{2}}=-\Omega_{c} \frac{\mathrm{d} x}{\mathrm{~d} t}+\omega_{c} \frac{\mathrm{d} z}{\mathrm{~d} t} \cos \Omega t-\frac{\Omega \omega_{c}}{2} z \sin \Omega t, \\
\frac{\mathrm{d}^{2} z}{\mathrm{~d} t^{2}}=\omega_{c}\left(\frac{\mathrm{d} x}{\mathrm{~d} t} \sin \Omega t-\frac{\mathrm{d} y}{\mathrm{~d} t} \cos \Omega t\right)+\frac{\Omega \omega_{c}}{2}(y \sin \Omega t+x \cos \Omega t) .
\end{gathered}
$$

Then we move from the laboratory frame $(x, y)$ to a rotating frame $(X, Y)$ through the transformation

$$
x+j y=(X+j Y) \exp j \Omega t,
$$

in order to obtain the following set of equations

$$
\begin{gathered}
\frac{\mathrm{d}^{2} X}{\mathrm{~d} t^{2}}-\Omega\left(\Omega+\Omega_{c}\right) X-\left(2 \Omega+\Omega_{c}\right) \frac{\mathrm{d} Y}{\mathrm{~d} t}+\frac{\Omega \omega_{c}}{2} z=0, \\
\frac{\mathrm{d}^{2} Y}{\mathrm{~d} t^{2}}-\Omega\left(\Omega+\Omega_{c}\right) Y+\left(2 \Omega+\Omega_{c}\right) \frac{\mathrm{d} X}{\mathrm{~d} t}-\omega_{c} \frac{\mathrm{d} z}{\mathrm{~d} t}=0, \\
\frac{\mathrm{d}^{2} z}{\mathrm{~d} t^{2}}+\frac{\Omega \omega_{c}}{2} X+\omega_{c} \frac{\mathrm{d} Y}{\mathrm{~d} t}=0 .
\end{gathered}
$$

The $\Omega^{2}$ factors are associated with the centrifugal force and the $2 \Omega$ terms with the Coriolis one. A classical normal mode analysis, with the amplitudes $\left(\boldsymbol{X}_{0}, \boldsymbol{Y}_{0}, \boldsymbol{z}_{0}\right)$ and the eigenfrequency $\omega=w \Omega_{c}$,

$$
[X(t), Y(t), z(t)]=\left(\boldsymbol{X}_{0}, \boldsymbol{Y}_{0}, z_{0}\right) \exp j \omega t,
$$

leads to the characteristic equation for the normalised eigenfrequency $w=\omega / \Omega_{c}$

$$
w^{6}-\left((1+u)^{2}+u^{2}+v^{2}\right) w^{4}+u^{2}\left((1+u)^{2}+\frac{3 v^{2}}{4}\right) w^{2}-\frac{1+u}{4} u^{3} v^{2}=0 .
$$

For stability, both the discriminant $D_{3}(u, v)$ of the associated algebraic third-order equation with unknown $w^{2}$ and the three real roots must be positive. Three real numbers are strictly positive if, and only if, their sum, product and sum of double products are strictly positive, this reduces the stability condition, $\omega$ real, to the relations:

$$
\begin{aligned}
D_{3}(u, v)= & u^{3}\left[2 u^{7} v^{2}+4 u^{7}+12 u^{6} v^{2}+20 u^{6}+u^{5} v^{4} / 16+57 u^{5} v^{2} / 2\right. \\
& +41 u^{5}+5 u^{4} v^{4} / 8+71 u^{4} v^{2} / 2+44 u^{4}-9 u^{3} v^{6} / 16 \\
& -21 u^{3} v^{4} / 16+21 u^{3} v^{2}+26 u^{3}-27 u^{2} v^{6} / 8 \\
& -79 u^{2} v^{4} / 8+3 u^{2} v^{2} / 2+8 u^{2}-7 u v^{8} / 16 \\
& -51 u v^{6} / 8-167 u v^{4} / 16-7 u v^{2} / 2+u \\
& \left.-v^{8}-3 v^{6}-3 v^{4}-v^{2}\right]>0 \\
F(u)= & u(1+u)>0
\end{aligned}
$$




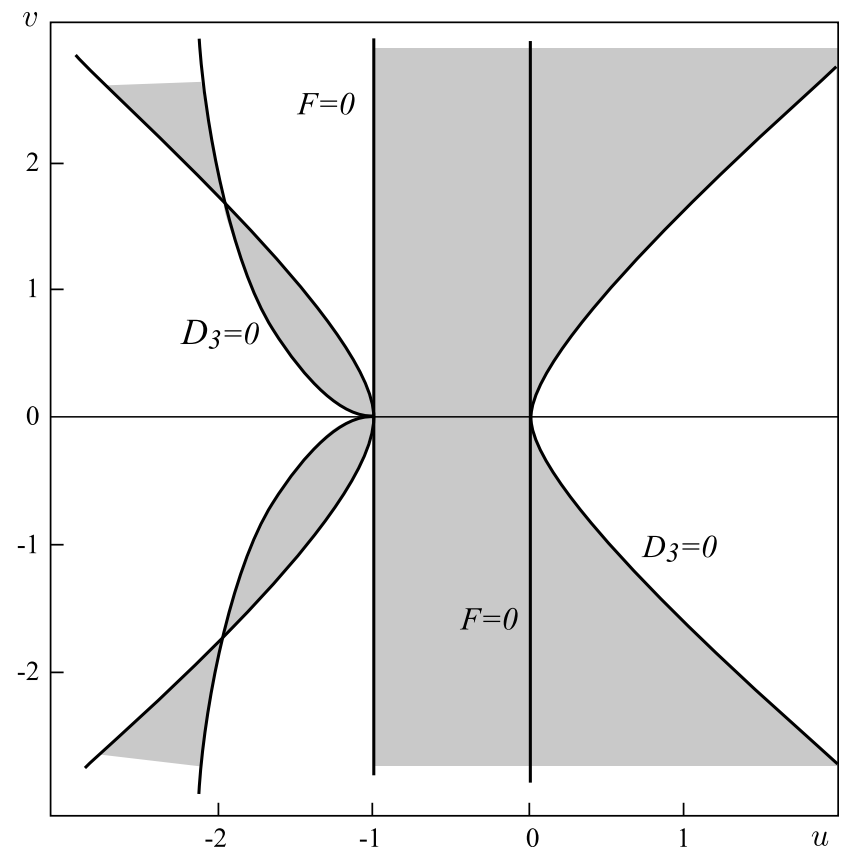

FIGURE 4. Stability regions, $D_{3}>0, F>0$, in the $u=\Omega / \Omega_{c}$ and $v=\omega_{c} / \Omega_{c}$ plane, for ion orbits in a rotating field generated by dephased orthogonal coils.

The shaded regions displayed in figure 4 are associated with unstable orbits in the ( $u=\Omega / \Omega_{c}, v=\omega_{c} / \Omega_{c}$ ) plane, the ion orbits remain confined and periodic outside these regions.

\section{Orbital stability with squirrel cage axial currents}

In the previous configuration, we have considered surface currents on two cylinders with axes perpendicular to the $z$ axis. Here, we consider axial surface currents on a cylinder whose axis is along the $z$ axis. These squirrel cage axial currents are along $z$ and distributed according to the relation $\cos (\theta-\Omega t)$ so that the resulting vector potential is given by $\boldsymbol{A}_{s}$. The dynamics of an ion is described by

$$
\begin{aligned}
\frac{\mathrm{d}^{2} x}{\mathrm{~d} t^{2}} & =\Omega_{c} \frac{\mathrm{d} y}{\mathrm{~d} t}-\omega_{c} \frac{\mathrm{d} z}{\mathrm{~d} t} \sin \Omega t, \\
\frac{\mathrm{d}^{2} y}{\mathrm{~d} t^{2}} & =-\Omega_{c} \frac{\mathrm{d} x}{\mathrm{~d} t}+\omega_{c} \frac{\mathrm{d} z}{\mathrm{~d} t} \cos \Omega t, \\
\frac{\mathrm{d}^{2} z}{\mathrm{~d} t^{2}} & =\omega_{c}\left(\frac{\mathrm{d} x}{\mathrm{~d} t} \sin \Omega t-\frac{\mathrm{d} y}{\mathrm{~d} t} \cos \Omega t\right)+\Omega \omega_{c}(y \sin \Omega t+x \cos \Omega t) .
\end{aligned}
$$

Moving from the laboratory frame $(x, y)$ to a rotating frame $(X, Y)$,

$$
x+j y=(X+j Y) \exp j \Omega t,
$$

we end up with the set of equations

$$
\frac{\mathrm{d}^{2} X}{\mathrm{~d} t^{2}}-\Omega\left(\Omega+\Omega_{c}\right) X-\left(2 \Omega+\Omega_{c}\right) \frac{\mathrm{d} Y}{\mathrm{~d} t}=0,
$$




$$
\begin{aligned}
\frac{\mathrm{d}^{2} Y}{\mathrm{~d} t^{2}}-\Omega\left(\Omega+\Omega_{c}\right) Y+\left(2 \Omega+\Omega_{c}\right) \frac{\mathrm{d} X}{\mathrm{~d} t}-\omega_{c} \frac{\mathrm{d} z}{\mathrm{~d} t} & =0, \\
\frac{\mathrm{d}}{\mathrm{d} t}\left(\frac{\mathrm{d} z}{\mathrm{~d} t}+\omega_{c} Y\right) & =0 .
\end{aligned}
$$

The $\Omega^{2}$ factors are associated with the centrifugal force and the $2 \Omega$ terms with the Coriolis one. Introducing $Y_{g}$, a constant of integration, a normal mode analysis with the eigenvectors $\left(\boldsymbol{X}_{0}, \boldsymbol{Y}_{0}, \boldsymbol{z}_{0}\right)$,

$$
\left[X(t), Y(t)-Y_{g}, z(t)\right]=\left(\boldsymbol{X}_{0}, \boldsymbol{Y}_{0}, z_{0}\right) \exp j \omega t,
$$

leads to the characteristic equation for the normalised eigenfrequency $w=\omega / \Omega_{c}$,

$$
w^{4}-\left((1+u)^{2}+u^{2}+v^{2}\right) w^{2}+u(1+u)\left(u+u^{2}-v^{2}\right)=0 .
$$

For stability, both the discriminant of the associated algebraic second-order equation with unknown $w^{2}$ and the two real roots must be positive. This discriminant is given by $(1+2 u)^{2}\left(1+2 v^{2}\right)+v^{4}$ and is obviously always positive, so the stability condition (two numbers are strictly positive if, and only if, their sum and product are strictly positive) reduces to $H F>0$, where $H=0$ is an hyperbola and $F=0$ is a set of two vertical lines

$$
\begin{aligned}
H(u, v) & =\left(u+\frac{1}{2}\right)^{2}-v^{2}-\frac{1}{4}, \\
F(u) & =u(1+u) .
\end{aligned}
$$

The eigenfrequencies $\omega$ of the periodic stables orbits $(H F>0)$ and of the growth rate $\gamma$ of the unstable escaping orbits $(H F<0$, shaded regions in figure 5), are given by the expressions:

$$
\begin{aligned}
& \sqrt{2} \omega= \pm \sqrt{\Omega^{2}+\left(\Omega_{c}+\Omega\right)^{2}+\omega_{c}^{2} \pm \sqrt{\left(\Omega_{c}+2 \Omega\right)^{2}\left(\Omega_{c}^{2}+2 \omega_{c}^{2}\right)+\omega_{c}^{4}}}, \\
& \sqrt{2} \gamma= \pm \sqrt{\Omega^{2}+\left(\Omega_{c}+\Omega\right)^{2}+\omega_{c}^{2}-\sqrt{\left(\Omega_{c}+2 \Omega\right)^{2}\left(\Omega_{c}^{2}+2 \omega_{c}^{2}\right)+\omega_{c}^{4}}} .
\end{aligned}
$$

In figure 5 the contour lines indicate the value of $H F$ which is proportional, for the escaping orbits, to the growth rate of the exponential instability.

\section{Principles of isotope separation with a rotating magnetic field}

On the basis of figures 3,4 and 5, we reach the important conclusion that the stability diagram of ion orbits in the $\left(u=\Omega / \Omega, v=\omega_{c} / \Omega_{c}\right)$ plane is very sensitive to the currents driving the fields for the very same magnetic field configuration $\omega_{c} \cos \Omega t \boldsymbol{e}_{x}+\omega_{c} \sin \Omega t \boldsymbol{e}_{y}+\Omega_{c} \boldsymbol{e}_{z}$. This sensitivity is due to the fact that, even if a superposition principle can be established in the rotating frame, the determinant of the sum of two matrices is different from the sum of their determinants, so the characteristic equation is expected to be very different for every new linear combination of the three basic potentials $\boldsymbol{A}_{s}, \boldsymbol{A}_{a}$ and $\boldsymbol{A}_{c}$, despite an interaction with the same rotating magnetic field.

This sensitivity to the electric part of the field offers opportunities to design dedicated configurations, but here, rather than addressing this optimisation issue, we 


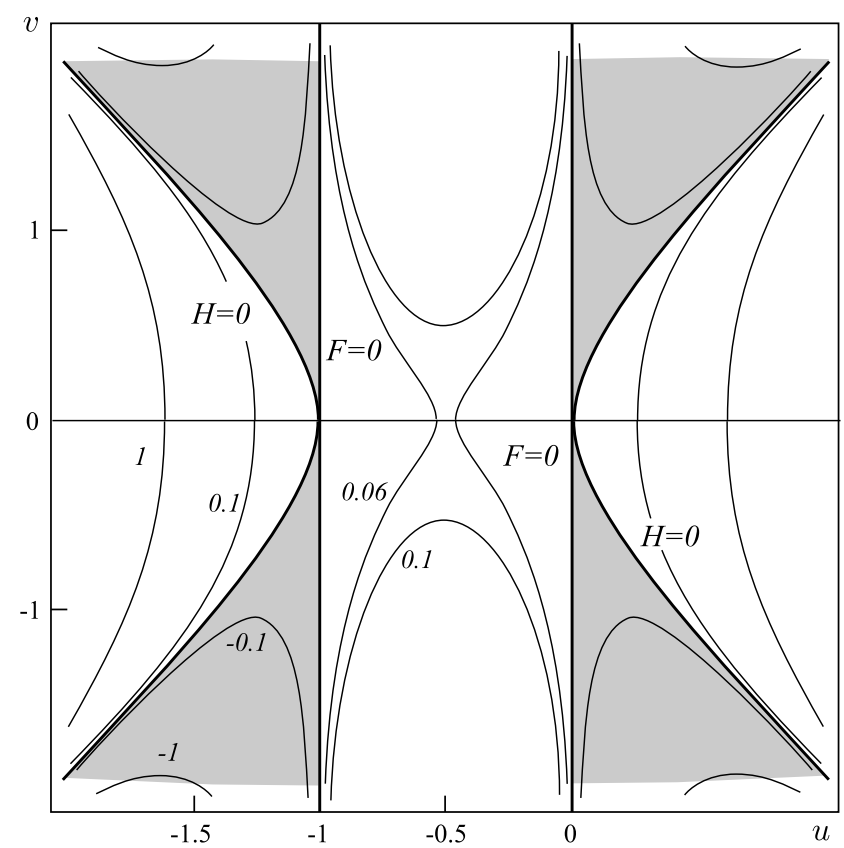

FIGURE 5. Stability regions, $H F>0$, in the $u=\Omega / \Omega_{c}$ and $v=\omega_{c} / \Omega_{c}$ plane, for ion orbits in a rotating field generated by squirrel cage axial currents; the contour lines are labelled with the value of $H F$.

will focus the study on the most simple diagram associated with squirrel cage currents along $z$, analysed and described in $\S 6$.

The case $\boldsymbol{A}_{s}$ has been investigated within the context of thermonuclear confinement and current generation but not for mass separation. In these studies, other normalised control parameters have been defined, different from $(u, v)$, and the emphasis was put on confined orbits. Here, for the purpose of studying the potential for mass separation, the choice of $u$ and $v$ is not only new, but particularly appropriate because $v$ is a structural parameter of the separator independent of the ion mass,

$$
v=\frac{\omega_{c}}{\Omega_{c}}=\frac{b}{B_{0}},
$$

and $u$ is a linear function of the mass. If we define the reference mass $m^{*}$ of the separator, $m^{*}=q B_{0} / \Omega$, then:

$$
u=\frac{\Omega}{\Omega_{c}}=\frac{m}{m^{*}} .
$$

The $(u, v)$ plane can thus be viewed as an $(m, b)$ plane. It is straightforward to understand that the various masses $m$ of an ion mixture, with lower and upper bounds $m_{1}<m_{2}$, will be distributed along a horizontal segment $v=b / B_{0}$ in between

$$
\frac{m_{1}}{m^{*}}<u<\frac{m_{2}}{m^{*}} .
$$

On the basis of this picture, illustrated in figure 6, the squirrel cage currents configuration $\boldsymbol{A}_{s}$ offers two different strategies for mass separation: (i) we can 
(a)

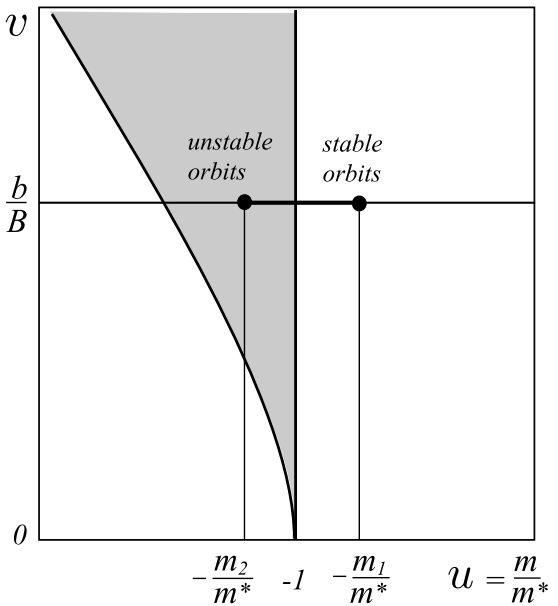

Light ions confined (b)

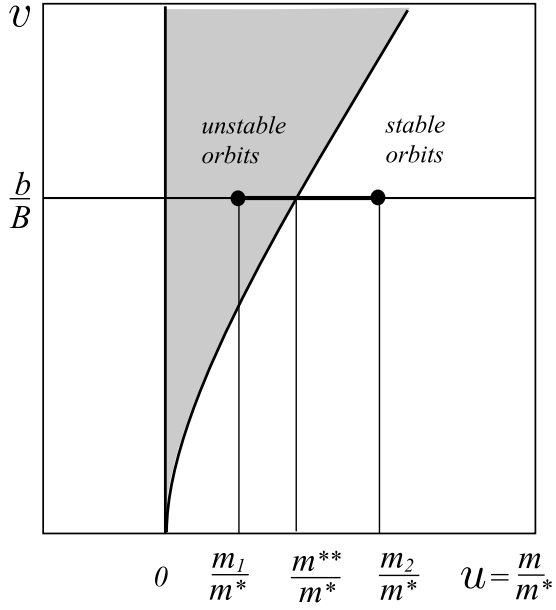

Heavy ions confined

FIGURE 6. Principles of rotating fields mass separation for a mass spectrum $m_{1}<m<m_{2}$ : (a) the light part is confined and the heavy one expelled or $(b)$ the heavy part of the spectrum is confined and the light one expelled.

confine the heavy part of the mass spectrum and expel the light one (figure $6 b$ ) or (ii) confine the light part and expel the heavy one (figure $6 a$ ). These two choices are associated with the fact that we have two choices for rotation $\pm \Omega \boldsymbol{e}_{z}$ for a given static field $B_{0} \boldsymbol{e}_{z}$, or two choices for the static field $\pm B_{0} \boldsymbol{e}_{z}$ for a given angular velocity $\Omega \boldsymbol{e}_{z}$. For the case $(b)$, in order to understand the separation process, the reference $m^{*}$ is to be supplemented by

$$
m^{* *}(b)=m^{*}\left(\sqrt{\frac{1}{4}+\frac{b^{2}}{B^{2}}}-\frac{1}{2}\right),
$$

as the curved boundary of the instability zone is given by the relation $m=m^{* *}(b)$. This principle of mass and isotope separation is the same for all the stability boundaries of figures 3-5 identified during the studies of the three basic cases $\boldsymbol{A}_{s}, \boldsymbol{A}_{a}$ and $\boldsymbol{A}_{c}$.

\section{Conclusions}

Despite these original promising conclusions, innovative mass separation with a rotating magnetic field remains an open problem of plasma physics and further studies are clearly needed.

This study was not intended to converge towards the final design of a mass separator for burned nuclear fuel management, but was aimed at advocating rotating plasma devices as promising candidates for closing the nuclear fuel cycle in next generation nuclear power plants (Fetterman \& Fisch 2011b; Timofeev 2014). In addition to a short review of existing concepts and results given in section two, new classes of field configurations and plasma processes for this strategic purpose were uncovered in this paper, and a rotating plasma driven by a rotating magnetic field was identified as a new candidate to solve the unsolved problem of plasma mass separation.

We showed that for a given rotating magnetic field $\boldsymbol{b}(t)$ there is an infinite number of associated inductive electric fields or vector potentials which cannot be reduced to 
a smaller set through a gauge transformation. These vector potentials can be obtained through any linear combination of: $\boldsymbol{A}_{s}$ and $\boldsymbol{A}_{a}$, or $\boldsymbol{A}_{s}$ and $\boldsymbol{A}_{c}$, or $\boldsymbol{A}_{c}$ and $\boldsymbol{A}_{a}$. An analysis of the orbital stability diagram for each of these basic fields was carried out, each of them presenting drawbacks and advantages with respect to the others. In light of this stability analysis, the general principles of mass separation using a rotating magnetic field were introduced using the field configuration $\boldsymbol{A}_{s}$ as an illustration.

The Alfvén standing wave field $\boldsymbol{A}_{a}$ seems to be a very promising candidate for mass separation since it is a normal mode of the plasma and does not suffer from plasma screening. However, this result is weakened by the fact that $\boldsymbol{A}_{a}$ is restricted to the vicinity of an electric node of the standing wave.

Although the two orthogonal dephased coils field $\boldsymbol{A}_{c}$ is probably the most widely used field configuration in synchronous and asynchronous rotating electric devices, the stability diagram associated with this configuration displays less opportunities for mass separation than the other two basic fields. Furthermore, the field penetration problem remains to be addressed in this configuration.

Finally, the squirrel cage axial currents configuration $\boldsymbol{A}_{s}$ exhibits the simplest stability diagram. Although screening effects are expected to disturb the stability diagram in this configuration, preliminary analysis seems to indicate that these effects could be overcome for typical processing plasma densities and dimensions. In addition, the nonlinearity brought by screening effects could, in some cases, be advantageous to design advanced separation schemes.

Further to these open questions, yet another challenge in the way of developing large-scale plasma separation lies in the extraction of ions after they have been separated. This question has been considered for other rotating plasma separators, but not answered in a fully satisfactory manner to date.

To conclude, we identified, described and analysed the principles, main advantages and drawbacks of a new kind of rotating plasma separator based on a rotating magnetic field. This new concept is free of the Doppler shift broadening which hinders classical ion cyclotron isotope separation methods (Dawson et al. 1976; Dolgolenko \& Muromkin 2009). Preliminary estimates suggest that rotating plasma separators could both satisfy the throughput requirement and be energetically attractive for spent fuel reprocessing applications. This novel approach could be extremely valuable for advanced closed nuclear fuel cycles. However, several challenges such as optimisation, screening and extraction remain to be addressed in order to design an industrial isotope and mass separator.

\section{Acknowledgement}

This work is dedicated to Professor N. J. Fisch, on the occasion of his 65th birthday, whose seminal contributions to numerous fields of plasma physics inspired these developments.

\section{REFERENCES}

Dawson, J. M., Kim, H. C., Arnush, D., Fried, B. D., Gould, R. W., Heflinger, L. O., Kennel, C. F., Romesser, T. E., Stenzel, R. L., Wong, A. Y. et al. 1976 Isotope separation in plasmas by use of ion cyclotron resonance. Phys. Rev. Lett. 37 (23), 1547-1550.

Dolgolenko, D. A. \& Muromkin, Y. A. 2009 Plasma isotope separation based on ion cyclotron resonance. Phys. Uspekhi 52 (4), 345-357.

Fetterman, A. J. \& FisCh, N. J. 2009 Wave-driven countercurrent plasma centrifuge. Plasma Sources Sci. Technol. 18, 045003. 
Fetterman, A. J. \& Fisch, N. J. $2011 a$ The magnetic centrifugal mass filter. Phys. Plasmas 18, 094503.

Fetterman, A. J. \& Fisch, N. J. $2011 b$ Metrics for comparing plasma mass filters. Phys. Plasmas 18, 103503.

Fetterman, A. J. \& Fisch, N. J. 2011c Wave-particle interactions in rotating mirrors. Phys. Plasmas 18, 055704-1.

Fisch, N. J. \& Watanabe, T. 1982 Field reversal by rotating waves. Nucl. Fusion 22 (3), 423-427.

Grossman, M. W. \& Shepp, T. A. 1991 Plasma isotope separation methods. IEEE Trans. Plasma Sci. 19 (6), 1114-1122.

Gueroult, R. \& Fisch, N. J. 2012 Practical considerations in realizing a magnetic centrifugal mass filter. Phys. Plasmas 19 (12), 122503-6.

Gueroult, R. \& Fisch, N. J. 2014 Plasma mass filtering for separation of actinides from lanthanides. Plasma Sources Sci. Technol. 23 (3), 035002.

Gueroult, R., Hobbs, D. T. \& Fisch, N. J. 2015 Plasma filtering techniques for nuclear waste remediation. J. Hazard. Mater. 297, 153-159.

Gueroult, R., RaX, J.-M. \& Fisch, N. J. 2014 The double well mass filter. Phys. Plasmas 21 (2), 020701.

Hole, M. J. \& Simpson, S. W. 1999 Separative performance in a vacuum arc centrifuges. IEEE Trans. Plasma Sci. 27 (2), 620-627.

Hugrass, N. W. \& Jones, I. R. 1983 The orbits of electrons and ions in a rotating magnetic field. J. Plasma Phys. 29 (1), 155-171.

Krishnan, M., Geva, M. \& Hirshfield, J. L. 1981 Plasma centrifuge. Phys. Rev. Lett. 46 (1), 36-38.

Milroy, R. D. 1999 A numerical study of rotating magnetic fields as a current drive for field reversed configurations. Phys. Plasmas 6 (7), 2771-2780.

Ohkawa, T. \& Miller, R. L. 2002 Band gap ion mass filter. Phys. Plasmas 9 (12), 5116-5120.

RaX, J. M., Fruchtman, A., Gueroult, R. \& Fisch, N. J. 2015 Breakdown of the brillouin limit and classical fluxes in rotating collisional plasmas. Phys. Plasmas 22 (9), 092101.

RAX, J.-M., Robiche, J. \& Fisch, N. J. 2007 Autoresonant ion cyclotron isotope separation. Phys. Plasmas 14 (4), 043102-8.

Shinohara, S., Nishida, H., Tanikawa, T., Hada, T., Funaki, I. \& Shamrai, K. P. 2014 Development of electrodeless plasma thrusters with high-density helicon plasma sources. IEEE Trans. Plasma Sci. 42 (5), 1245-1254.

Soldatenkov, T. R. 1966 Motion of charged particles in rotating and constant magnetic fields. Sov. Phys.-Technical Phys. 11 (2), 179-181.

Timofeev, A. V. 2007 Plasma method for processing spent nuclear fuel. Plasma Phys. Rep. 33 (11), 890-905.

Timofeev, A. V. 2014 On the theory of plasma processing of spent nuclear fuel. Phys. Uspekhi 57 (10), 990-1021. 